\title{
WIND VARIABILITY IN O-TYPE STARS
}

\author{
H.F. HENRICHS, L. KAPER \\ Astronomical Institute "Anton Pannekoek", University of Amsterdam, \\ and Center for High-Energy Astrophysics, \\ Kruislaan 403, 1098 SJ Amsterdam, Netherlands \\ and \\ J.S. NICHOLS \\ Science Programs, Computer Sciences Corporation, 10000-A Aerospace Rd., \\ Lanham-Seabrook, MD 20706, U.S.A.
}

\begin{abstract}
The search for the cause of the discrete absorption components (DACs) in $P$ Cygni profiles in UV spectra of $O$ stars continues: pulsation, rotation and magnetic fields are probably all in various degrees important. We highlight some key observations which show that DACs start close to the stellar surface, and emphasize the possible role of localized magnetic fields (which are still to be detected).
\end{abstract}

\section{Introduction}

Discrete absorption components (DACs) in P Cygni lines in UV spectra of $\mathrm{O}$ and $B$ stars were first detected more than 15 years ago, but their origin is still uncertain. They are readily recognized in individual spectra as 'unexpected' absorption features at high velocities $(-1000$ to $-2000 \mathrm{~km} / \mathrm{s})$ in stellar wind lines (N V, C IV, Si IV in O and early B stars, and Si III, N IV, C III, Al III in intermediate to late $B$ stars, e.g. Henrichs 1988). The phenomenon that produces DACs is clearly a fundamental property of the winds of early-type stars. Estimates of the frequency of DACs approach $90 \%$ for the $\mathrm{O}$ stars and B supergiants (Prinja and Howarth 1986), whereas among the nonsupergiant B stars, DACs uniquely occur in Be stars (Henrichs 1984) with a frequency of at least $65 \%$ (Grady et al. 1987, Prinja 1989). DACs vary in strength and velocity, with a typical recurrence timescale of a few days in $\mathrm{O}$ stars, during which a DAC develops and migrates to an asymptotic velocity, while decaying in strength on a typical timescale of a week. In the meantime a new DAC develops, with a similar (but not identical) pattern. The best examples are the O7.5 III stars $\xi$ Per (Prinja et al. 1987, Henrichs et al. 1994, hereafter HKN) and 68 Cyg (Prinja and Howarth 1989) and $\zeta$ Pup O4I(n)f (Prinja et al. 1992). Figure 1 gives examples of rapidly and slowly developing DACs. Many other examples can be found in Kaper (1993).

While significant theoretical progress has been made regarding the properties of radiatively driven flows (Owocki et al. 1988, Owocki 1991, Puls et al. 1993, Owocki, this volume) there is still no answer to the question why a DAC should develop at the first place, in particular, no prediction can be made when, and under which circumstances a DAC should begin. DACs 


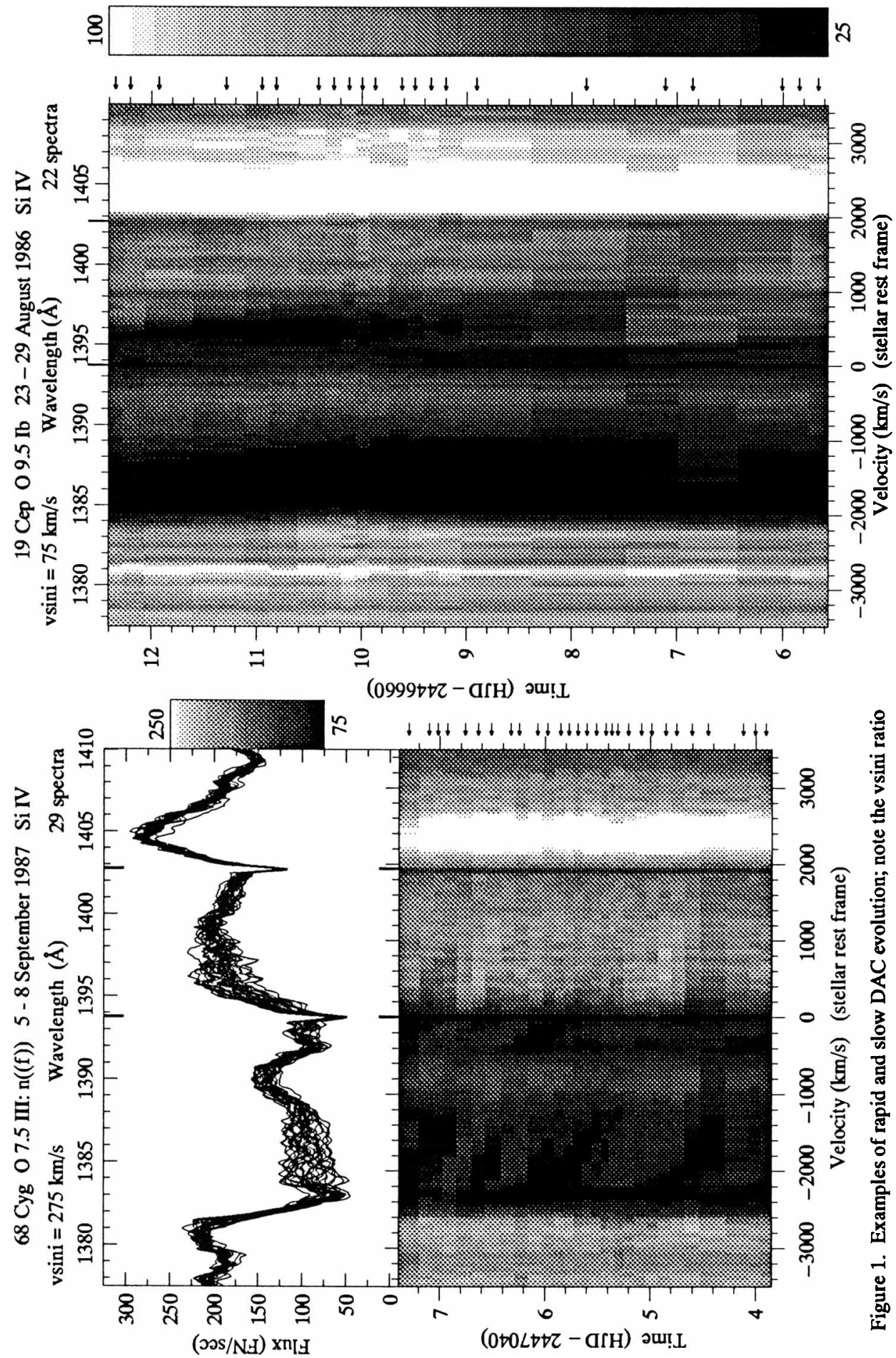


may form as a necessary consequence of the intrinsically unstable nature of such winds (Owocki c.s.), or, alternatively, DACs might start to develop as the result of some changing condition at or near the stellar surface (our working hypothesis, e.g. Kaper et al. 1992, HKN, Kaper et al. 1994). The real question is actually whether the second alternative is needed in addition to the first.

In this review we concentrate on a number of recent observations which all suggest that the outflowing material which is ultimately observed as DACs, can be already identified close to the stellar surface. We first show that the DAC variability is closely associated with variations in the lowvelocity and highest-velocity regimes of variable wind lines where no DACs are observed. Secondly, we present some coordinated observations of optical ( $\mathrm{H} \alpha$ and $\mathrm{He}$ II) and UV wind lines, which suggest that, at least in some $\mathrm{O}$ stars, the beginning of a DAC coincides with the appearance of additional blue emission in the optical lines. Finally we discuss a simplified model in which DACs arise from inhomogeneities at the base of the wind, caused by the (hypothesized) presence of weak-magnetic field configuration(s), which temporarily trap some of the wind material on its way out. The observed temporal behavior of the optical lines is in first approximation consistent with such a model.

\section{DACs, blue-edge and low-velocity variability}

Since the launch of the IUE satellite in 1978, about a dozen $O$ stars have been observed intensively in the UV spectral region with a typical time resolution of one to several hours, resulting in a number of very well planned time series, which show remarkable variety in P Cygni line variability. Three forms of variability are usually distinguished:

(1) the DACs themselves, mostly observed in unsaturated lines, in particular Si IV.

(2) in the blue-edge of saturated P Cygni lines (C IV, N V). The whole edge is observed to significantly shift about $10 \%$ in velocity, on a typical timescale of hours to days. The highest velocity of the black bottom of the profile remains approximately at the same position. Figure 2 shows a detailed example for the star $\xi$ Per.

(3) in the low-velocity regime, below $500 \mathrm{~km} / \mathrm{s}$, especially in the unsaturated subordinate line N IV $\lambda 1718$ (see figure 3 (from HKN)).

It is now clear that these three forms are all associated with the behavior of DACs. The precise phase relation, however, between the high-velocity edge and the DACs has not been unraveled for all stars. Figure 2 shows the parallel behavior of the Si IV edge, where DACs dominate the variable behavior, and the strikingly corresponding behavior of the C IV edge, which show that they must be related in origin. Figure 3 shows the parallel behavior 


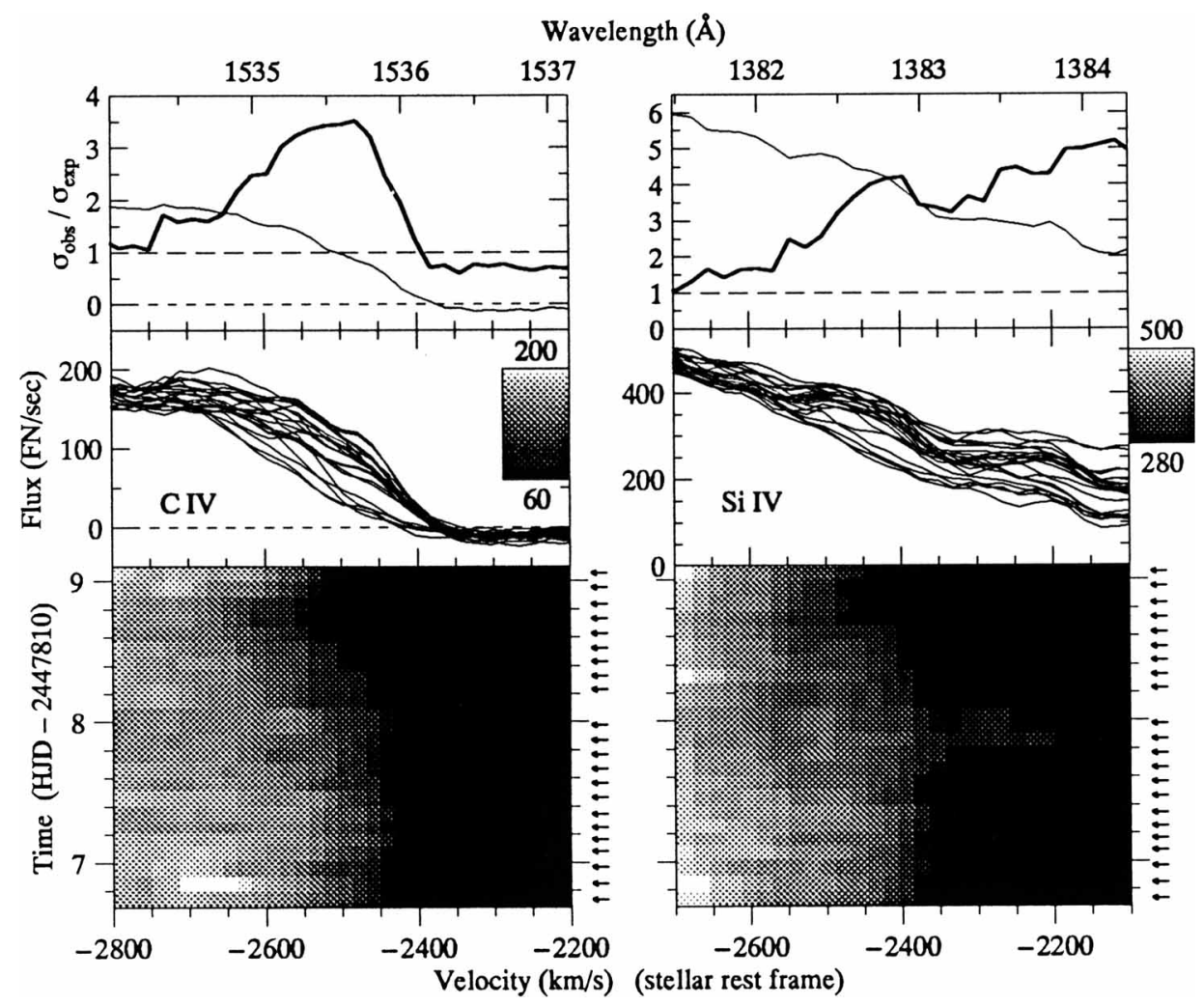

Fig. 2. $\xi$ Per October 1989. The strikingly parallel behavior of the edges of the saturated $\mathrm{C}$ IV line and the unsaturated Si IV line in which DACs dominate the variability, shows that the blue-edge variability in saturated lines is caused by DACs with a high optical depth

of the low-velocity N IV region and the DACs in Si IV: again a one-to-one correspondence of the phenomena.

It is worth noting that a quantification of the significance of variability in high-resolution IUE spectra, with a $\mathrm{S} / \mathrm{N}<30$, has proven to be crucial to obtain these results. This problem has been satisfactory solved by HKN: a two-parameter fit of the form $A \tanh (B / F)$ to the observed signal to noise ratio as a function of flux level $F$ has been applied to the statistically rigorous method developed by Fullerton (1990). The upper panels in figures 2 and 4 show the ratio of the observed to the expected variability, which measures the significance of the variations. 

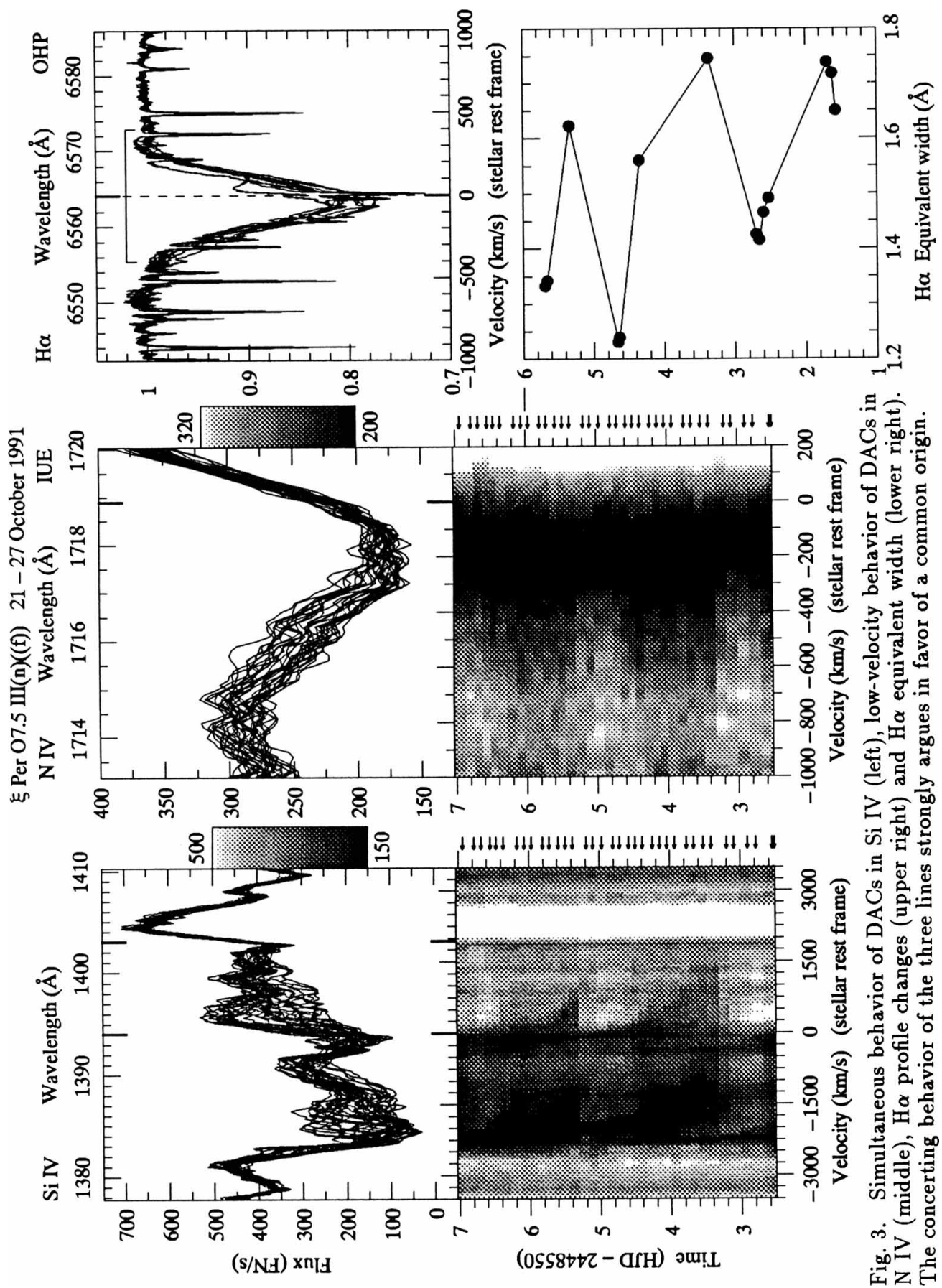


\section{Simultaneous low- and high-velocity stellar wind observations}

A strong argument that wind variability in the form of O-star DACs originates close to the photosphere is that DACs in the Si IV resonance lines develop at the same time when enhanced absorption appears at low velocity $(<250 \mathrm{~km} / \mathrm{s})$ in the subordinate N IV $\lambda 1718$ line (figure 3) (HKN, Kaper and Henrichs 1994). This N IV line can only be formed close to the star, because the strength of subordinate lines decreases with (density) ${ }^{2}$, which is unlike the resonance lines, where the strength decreases proportionally to density and which are therefore observed throughout the wind (see HKN).

Further evidence that DACs have their origin very near the star comes from the observation that rotation plays a significant role. Starting with Prinja (1988) and Henrichs et al. (1988), all papers in which more than one sequence of DACs was described, strongly suggest that DAC patterns repeat on the rotational timescale of the star (see also Prinja, this volume). Fig. 4 illustrates this over a 4 -year period for $\xi$ Per (rotation period 2 to 4 days).

We have observed three cases by means of simultaneous optical and UV spectroscopy, which strongly suggest that matter which piled up at a certain confined location close to the photosphere, is carried around by rotation. This matter is observable as additional $\mathrm{H} \alpha$ or He II 4686 emission as soon as it appears around the limb of the star. Pulsation cannot cause such an effect, because the pulsational time scale is too short to account for the observed changes. The three cases are:

(1) In $\xi$ Per not only the equivalent width of the $\mathrm{H} \alpha$ absorption line varies in concert with the DACs (figure 3, right), but the additional emission appears at the blue side of the profile (at about $-v \sin i$ ) and moves towards the red (figure 5a). This happened during 3 subsequent DAC events.

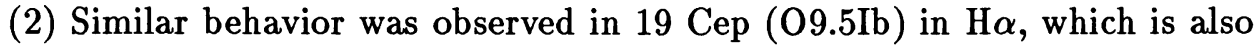
in absorption in this star. Just prior to the epoch that a DAC started, additional emission appeared on the blue wing of the line and moved to the red (Kaper et al. 1994).

(3) In the O6I(n)fp star $\lambda$ Cep simultaneous variations were observed at the blue side of the He II 4686 line $(<200 \mathrm{~km} / \mathrm{s}$ ) and the onset of DACs (figure 5b, Henrichs 1991).

It looks as if the beginning of a DAC can be predicted from the rather sudden appearance of additional blue emission in the optical lines. This result has obviously to be confirmed for other stars. The observations above are consistent with a configuration in which a part of the stellar wind with enhanced density at a given location just above (because of the emission) the stellar surface is carried into the line of sight by the rotation of the star (because of the movement from blue to red). This location is likely the footpoint of a DAC that will appear somewhat later, depending on the rotational and flow timescale. 

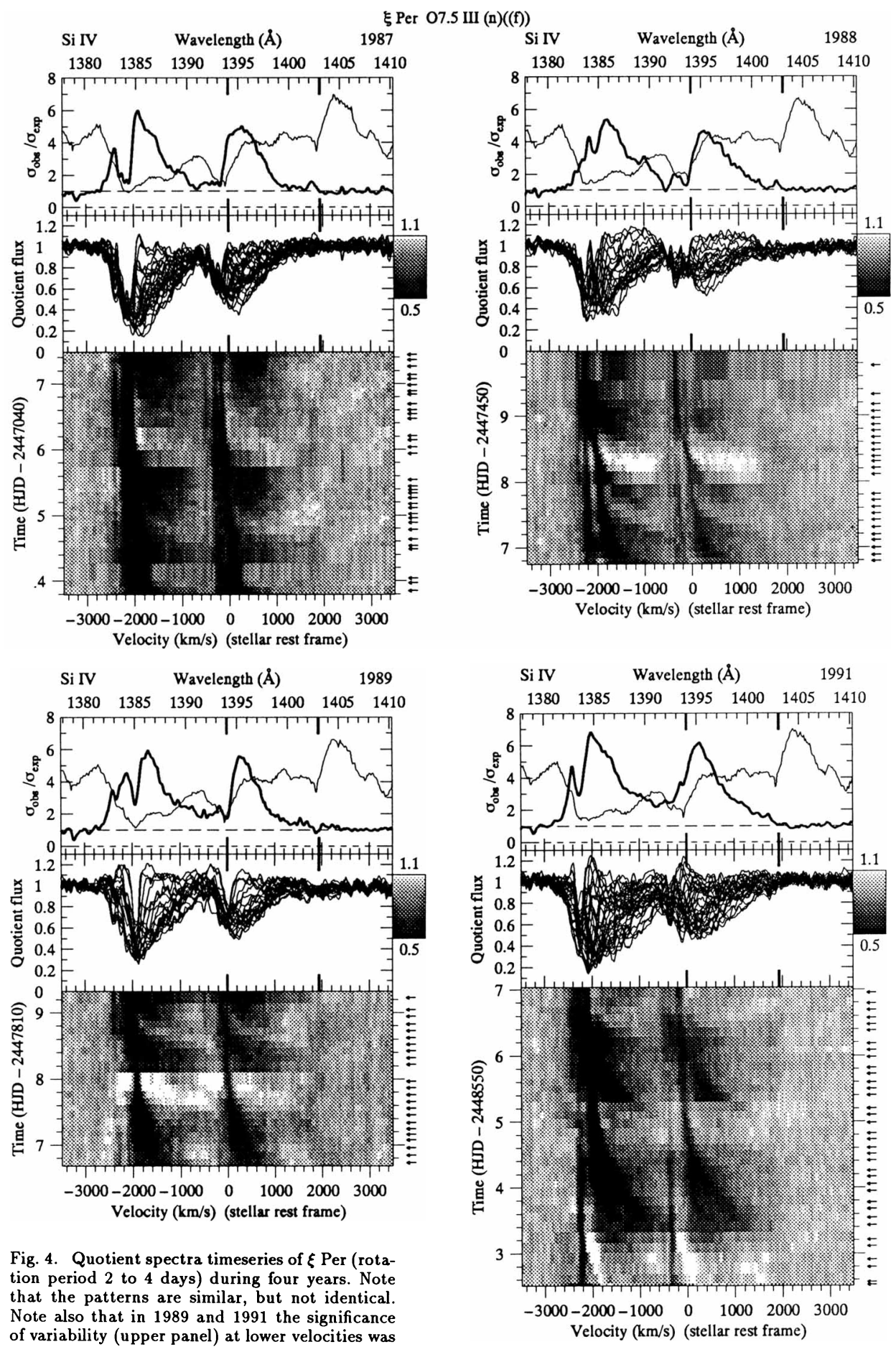

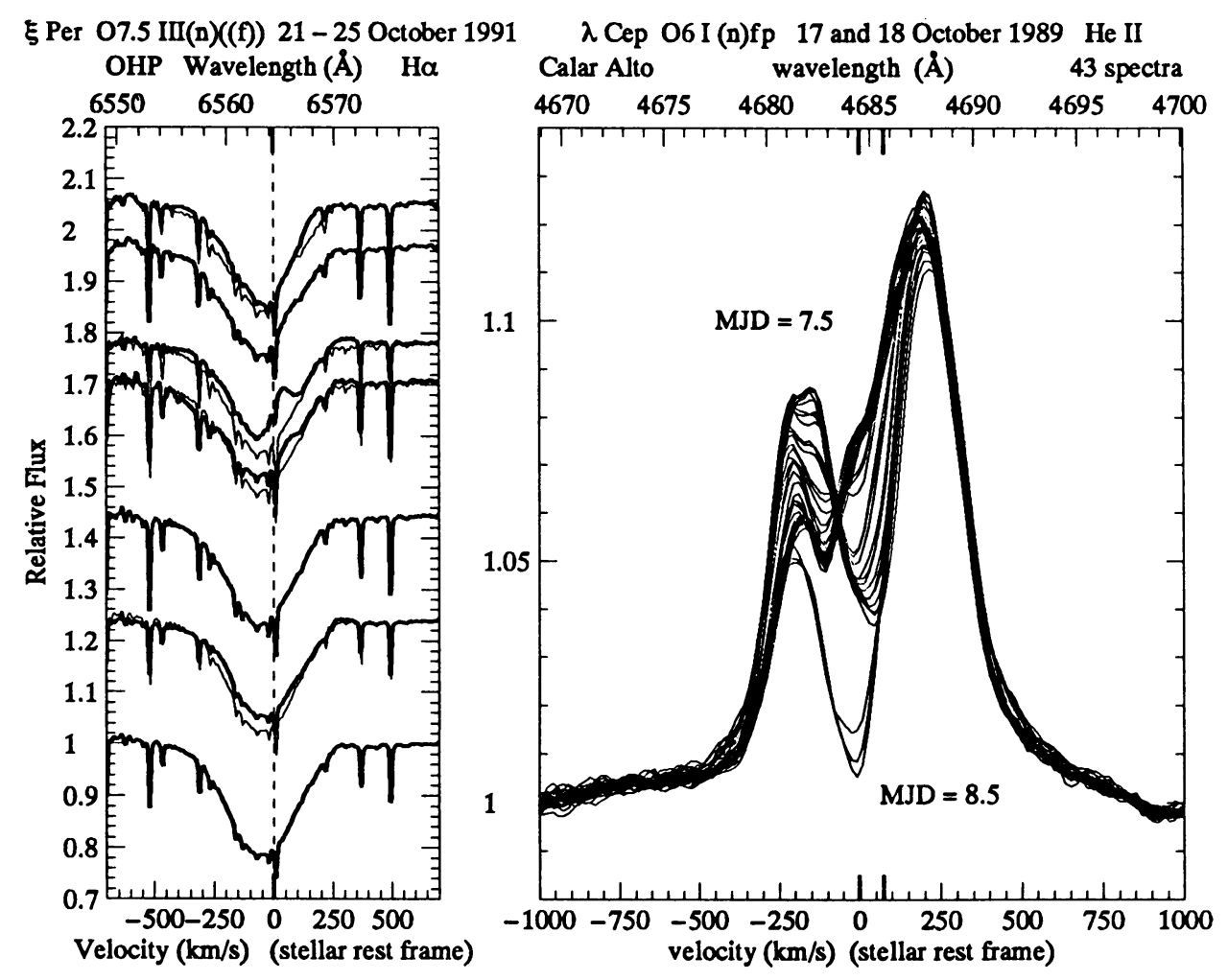

Fig. 5. a. left: Representative H $\alpha$ spectra of $\xi$ Per in October 1991, in the vertical direction shifted proportionally with time. The first spectrum in each of the three episodes of figure 3 is overplotted with a thin line, in order to demonstrate the appearance of additional emission at the blue side of the profile just prior to the beginning of a Si IV DAC.

b. right: Sample profiles He II $\lambda 4686$ of $\lambda$ Cep in October 1989, during two consecutive nights. Typical time resolution was $20 \mathrm{~min}$. On top of the asymmetric double-peaked emission line, there is additional emission appearing at $-200 \mathrm{~km} / \mathrm{s}$, which corresponds to $-v \sin i$, which was rapidly followed by additional central absorption. The next day, the emission was gone.

\section{DACs, rotation and magnetic fields in $O$ stars}

The considerations above argue in favor of an interpretation that DACs originate at a particular spot on the stellar surface. As put forward by HKN and Kaper et al. (1994) the most likely explanation for such behavior is the presence of a localized magnetic field, which traps some fraction of the outflowing wind, and makes a non-axisymmetric wind, which leads in turn to DACs, like in a modified model of corotating interacting regions (CIRs, Mullan 1984). It could also be that simply the fact that the lower boundary 
condition of the wind at such a magnetic patch is different from elsewhere, is sufficient to create an inhomogeneous wind which, according to its unstable nature, form structures which are observed as DACs. Anyhow, the above given argumentation leads automatically to the next logical step, namely to investigate whether magnetic fields are actually present in $\mathrm{O}$ stars.

Till recently, only null detections with $1 \sigma$ of order $100 \mathrm{G}$ existed for two $O$ stars ( $\zeta$ Pup, Barker et al. 1981, and $\zeta$ Oph, Barker et al. 1985), but only two or three measurements were obtained. Currently a large long-term survey for magnetic fields in $\mathbf{O}$ stars is being carried out by Bohlender (this volume) on a variety of telescopes with the UWO Balmer line polarimeter. To date only upper limits of about $300 \mathrm{G}$ has been established. Also for these objects usually one measurement was obtained, and none of our targets has been included.

With a field configuration as suggested above, it is expected that the longitudinal component of the magnetic field will be modulated with the rotation of the star. This means that in case of a dipolar type field only twice per rotation the maximum field strength can be measured. Single snapshots are therefore unlikely to be obtained during optimum orientation, and longer sampling is needed to establish the presence of a magnetic field.

From the theoretical side, it is difficult to estimate the strength of such a field (see e.g. Moss, this volume), although Maheswaran and Cassinelli $(1988,1992)$ were able to set constraints. That magnetic fields should exist in $O$ stars is shown by the existence of the very strong fields at the end of their evolution in the form of collapsed neutron stars. An upper limit of about $100 \mathrm{G}$ is set by the requirement that a stronger field would cause a rapid spin down of the star during its main-sequence lifetime (MacGregor et al. 1992), which is not the case, as many evolved stars are still moderate rotators.

About the configuration and stability of such weak fields, not much is known. If the wind modulation in $\mathrm{O}$ stars, as illustrated in figure 4, indeed reflects the presence of surface fields, it is clear that they are not very stable, with a lifetime of the order of a month, i.e. 10 stellar rotations.

\section{Discussion and prospects}

Although some progress has been made, we are still far from understanding the different roles played by rotation, non-radial pulsation (undoubtedly present) and magnetic fields (yet to be detected) and their mutual interaction with respect to the behavior of $\mathrm{O}$-star winds. It is important to stress that we have not found direct observational evidence that stellar pulsations affect the wind variability. A tentative picture could be summarized as follows: the non-radial pulsation causes the instabilities in the wind to develop. At places where at the surface a magnetic field "patch" is able 
to influence the flow behavior, the lower boundary condition of the wind will be different. We observe the less-accelerated, denser material just above the surface in the optical lines, which will be rotationally modulated. The non-axisymmetric flow with contrasting velocity fields (i.e. of the normal wind and above a patch) gives rise to the development of CIR's (like in the solar wind case), which in turn gives rise to density enhancements, which we observe as DACs. It is clear that the crucial test of such a model should have to come from simultaneous UV spectroscopy and magnetic-field measurements. $\mathrm{F}$ rom the theoretical side it would be interesting to investigate the existence and stability of magnetic patches as described above. Finally, much progress is expected from hydrodynamical simulations and line profile calucations by Owocki, Puls and others.

\section{Acknowledgements}

The authors are very grateful to their many co-authors and co-observers on various optical telescopes around the globe, especially H. Ando, H. Barwig, K. Bjorkman, A. Fullerton, D. Gies, R. Hirata, E. Kambe, D. McDavid. L. Snoek, H. Volten, R. Zwarthoed and others. Discussions with S. Owocki and D. Bohlender are also gratefully acknowledged. This work was partially supported by NASA grant NAS5-31835 and by the Netherlands Organization for the Advancement of Pure Research (NWO).

\section{References}

Barker, P., Landstreet, J., Marlborough, J., Thompson, I., Maza, J.: 1981, Astrophys. J. 250, 300

Barker, P., Landstreet, J., Marlborough, J., Thompson, I.: 1985, Astrophys. J. 288, 741

Fullerton, A.W., 1990, Thesis, University of Toronto

Grady, C.A., Bjorkman, K.S., Snow, T.P.: 1987, Astrophys. J. 320, 376

Henrichs, H.F.: 1984, in ESA, ed., Fourth European IUE Conference, Roma, Italy, ESA SP-218, 43.

Henrichs, H.F.: 1988, in P.S. Conti and A.B. Underhill, eds., NASA/CNRS monograph series, NASA SP-497, 199.

Henrichs, H.F.: 1991, in Baade, D., ed., Rapid Variability of OB-stars: Nature and Diagnostic Value, ESO: Garching, 199.

Henrichs, H.F., Gies, D.R. Kaper, L., Nichols-Bohlin, J.S., et al.: 1990, in ESA, ed., Evolution in Astrophysics: IUE Astronomy in the era of new space missions, ESA SP-310, 401.

Henrichs, H.F., Kaper, L. and Zwarthoed, G.A.A.: 1988, in ESA, ed., Celebratory Symp.: $A$ decade of $U V$ astronomy with IUE, ESA SP-281, Volume 1, 145.

Henrichs, H.F., Kaper, L. and Nichols, J.S. : 1994, Astron. Astrophys. 265, 685 (HKN)

Howarth, I.D., Prinja, R.K. : 1989, Astrophys. J. Suppl. 69, 527

Kaper, L., 1993, Thesis, University of Amsterdam

Kaper, L., Henrichs, H.F., Zwarthoed, G.A.A. and Nichols-Bohlin, J.: 1990, in L. A. Willson, G. Bowen and R. Stalio, eds., NATO Workshop on Mass Loss and Angular Momentum of Hot Stars, Kluwer, 213 
Kaper, L., Henrichs, H.F., Nichols-Bohlin, J.S.: 1992, in B. Warner, ed., Variable Stars and Galaxies, Cape Town, Feb., ASP Conference Series, 135.

Kaper, L., Henrichs, H.F.: 1994, in A. Moffat, S. Owocki, A. Fullerton , N. St.-Louis, eds., Workshop on instability and outflows from stars, ASP Conference Series,

Kaper, L., Henrichs, H., Ando, H., Bjorkman, K., Fullerton, A., Gies, D., Hirata, R., Kambe, E. McDavid, D., Nichols, J.: 1994, Astron. Astrophys. submitted

MacGregor, K.B., Friend, D.B., Gilliland, R.L.: 1992, Astron. Astrophys. 256, 141

Maheswaran, M., Cassinelli, J.P.: 1988, Astrophys. J. 335, 931

Maheswaran, M., Cassinelli, J.P.: 1992, Astrophys. J. 386, 695

Mullan, D.: 1984, Astrophys. J. 283, 303

Owocki, S, Castor, J., Rybicki, G.: 1988, Astrophys. J. 335, 914

Owocki, S.: 1991, in I. Hubeny and L. Crivellari, eds., NATO Workshop on Stellar Atmospheres: Beyond Classical Models, Kluwer,

Prinja, R.K.: 1988, Mon. Not. Roy. Astr. Soc. 231, 21P

Prinja, R.K.: 1989, Mon. Not. Roy. Astr. Soc. 241, 721

Prinja, R.K. and Howarth, I.D.: 1986, Astrophys. J. Suppl. 61, 357

Prinja, R.K. and Howarth, I.D.: 1988, Mon. Not. Roy. Astr. Soc. 233, 123

Prinja, R.K., Howarth, I.D. and Henrichs H. F.: 1987, Astrophys. J. 317, 389

Prinja, R.K., et al.: 1992, Astrophys. J. 390, 266

Puls, J, Owocki, S., Fullerton, A.: 1993, Astron. Astrophys. 279, 457

\section{Discussion}

Prinja: It is not clear to me in your CIR picture, what the relation is between high-velocity blue-edge changes in the saturated UV P Cygni profiles and the (apparently connected) development of DACs at very low velocity.

Owocki (response to Prinja): The fact that the C IV blue edge is gradual despite the strong opacity of this resonance line has two possible interpretations. First is that it arises from very low-density, high-speed flow, such as seen in the wind instability simulations. A second possibility is that it arises from a high-speed stream forming from a rapidly diverging $B$ field, relatively close to the star, but geometrically narrow so that it occults only a small fraction of the stellar disk. We hope soon to compute a dynamical "CIR" model to see if this idea can be made to match observations.

Lafon: For corotating interacting regions to organize, it is necessary that the magnetic field be frozen in the matter (as in the solar wind). This seems difficult for weak fields, in cool disks, with high density. The validity of the assumption of "quasi-frozen field" (infinite conductivity) must be verified. Maybe this could be applied with better results for hot stars than for Be stars.

Henrichs: I agree, and would like to emphasize that DACs in Be stars are likely to have a different origin then in $\mathbf{O}$ stars.

Dachs: Similar DAC development might also be visible in spectra of the Be star HR 2855 (FY CMa) obtained in early 1987, when DACs were visible 
in Si IV and C IV profiles taken by IUE at about the same time when He I $\lambda 5876 \AA$ showed inverse P Cygni structure.

Henrichs: Thank you for mentioning this. It would be interesting to follow the DACs in this star with a high time resolution. As far as I know that has not been done.

Le Contel: How can you predict the appearance of the DACs? I did not understand it. Similar DACs appear in some $\beta$ CMA stars associated with the phase of the pulsation. It would be interesting to confront the two phenomena.

Henrichs: We found that the beginnings of DACs in $O$ stars can be predicted from the the rather sudden appearance of additional emission at the blue side of $\mathrm{H} \alpha$ in the cooler $\mathrm{O}$ stars, and of $\mathrm{He}$ II in the hotter $\mathrm{O}$ stars. I don't know whether this behavior is also observed in the $\beta$ CMA stars you mention. It should be looked at.

Smith: You mentioned that there was no observational evidence for your magnetic patch model for $\mathrm{O}$ stars, but in fact if you look carefully at the original paper suggesting NRP in an Oe star, Vogt and Penrod (1983) monitored lpv's of two He I profiles, $\lambda 6678$ and $\lambda 4471$, in $\zeta$ Oph. What was left understated in their paper was rather significant differences in the lpv's of these two lines. At that time neither Don Penrod, nor I could understand this. However, the differences in behavior that I described yesterday for $\lambda$ Eri between $\lambda 6678$ and $\lambda 4922$ (the triplet analogy of $\lambda 4471$ ) may perhaps be in a rather similar way to what I suggested therefore. Therefore there are some grounds for suspecting that magnetic activity may be present as well in $\zeta$ Oph.

Henrichs: I hope that such fields will be searched for soon.

Harmanec: I have three questions:

(1) When I was arguing in favour of local outflow of material from the equator of rapidly rotating stars in my 1991 Garching review, I pointed out that there should be some logical time delay in variations detected in lines formed close to, and far from the photosphere. Can you see evidence of such time delays in your data?

(2) Do you need the local magnetic field to start the outflow? Can it not start from a high wave crest of NRP, where the apparent irregularity could be related to multiperiodicity of NRP waves?

(3) It seems to me that the periodogram for $\lambda$ Cep you have shown indicates that there may be a systematic variation in the value of the period accross the line (differential rotation??) What do you think about that?

Henrichs: (1) We have not searched for such time delays in different optical lines. We have data for a few stars which possibly carry this information. 
(2) The magnetic field is not needed for the outflow to start. Above a magnetic patch, it rather slows down the outlow. If I look at figure 4, the best evidence for long-term behavior we have sofar, it is clear that there is no obvious long-term clock driving the DAC patterns. This suggests that periodic phenomena like (multiperiodic) NRP is unlikely the trigger. This is why we propose short-lived magnetic patches: if they are present, they will be carried around by rotation, but if they disappear and reappear later, the phase is lost.

(3) We have not finished our period analysis for this star, as this is nontrivial. It is an interesting suggestion, which we should investigate.

Baade: Is it possible that the difference in apparent predictability between $\mathrm{O}$ stars and Be stars concerning the development of DACs arises from the difference in the ratio of wind flow time to rotation period? If a possible circumstellar pattern gets wound up by rotation, it may be difficult to see correlations.

Henrichs: This is certainly possible, but more factors are likely involved. For instance the observed correlation between $V / R$ variability in Be stars and the strength of the DACs (Doazan et al, Telting et al) suggests that DACs in these stars are related to the conditions in the disk, which is also very different from what we see in $\mathrm{O}$ stars. 